\title{
A PRÁTICA CLÍNICA DO FARMACÊUTICO NO NÚCLEO DE APOIO À SAÚDE A FAMÍLIA
}

\author{
CLINICAL PRACTICE OF PHARMACISTS IN FAMILY HEALTH \\ SUPPORT TEAM
}

\section{LA PRÁCTICA DEL FARMACÉUTICO CLÍNICO EN EL EQUIPO DE SOPORTE PARA LA SALUD DE LA FAMILIA}

\author{
Daniela Álvares Machado Silva ${ }^{1}$ \\ Simone de Araújo Medina Mendonça² \\ Djenane Ramalho de Oliveira ${ }^{3}$ \\ Clarice Chemello ${ }^{4}$
}

Resumo Este estudo teve como objetivo compreender os elementos essenciais do processo de sistematização da prática clínica de uma farmacêutica da atenção primária à saúde com base no referencial teórico-metodológico da atenção farmacêutica, que subsidia o serviço clínico de gerenciamento da terapia medicamentosa. Tratouse de pesquisa qualitativa autoetnográfica, construída de forma colaborativa entre os autores, de outubro de 2014 a outubro de 2015, nos Centros de Saúde da Prefeitura Municipal de Belo Horizonte, onde uma das autoras trabalha. Os dados foram produzidos por meio de observação participante, diários de campo, reflexões e entrevistas semiestruturadas com farmacêuticos que desenvolviam prática clínica na atenção primária à saúde. Os resultados evidenciaram que os principais elementos para sistematização da prática clínica passam pela 'construção de uma nova identidade profissional na equipe multiprofissional' e pela 'incorporação de novas atividades na rotina de trabalho' que, combinadas, resultam em uma 'proposta de integração de um serviço de gerenciamento da terapia medicamentosa nos fluxos das unidades de saúde'. Dessa forma, para que o farmacêutico possa legitimar o seu papel no cuidado do paciente, é preciso mudar, transformar, reorganizar e reconstruir a sua prática.

Palavras-chave autoetnografia; atenção primária à saúde; gerenciamento da terapia medicamentosa; atenção farmacêutica.
Abstract The aim of this study was to understand the essential elements of the systematization process of the clinical practice of a pharmacist in primary health care. This systematization was based on the framework of pharmaceutical care practice, which provides the foundation for comprehensive medication management services. The methodology utilized was autoetnography, built collaboratively between the authors. The data have been produced through participant observation, field journals, reflections and semi structured interviews with pharmacists who are building clinical practices in primary health care. The results have demonstrated that the main elements associated with the systematization of clinical practices are the construction of a new professional identity in a multiprofessional team' as well as 'the incorporation of new activities in the work routine', that, combined, result in 'Integration of comprehensive medication management services in the flow of the health unit'. In this way, in order for the pharmacist to legitimize his role in patient care, it is necessary to change, to transform, to reorganize and to rebuild his practice.

Keywords auto ethnography; primary health care; comprehensive medication management; pharmaceutical care. 


\section{Introdução}

A atenção farmacêutica é um arcabouço teórico que guia a prática clínica do farmacêutico e é ofertada no sistema de saúde como um serviço denominado gerenciamento da terapia medicamentosa (GTM) (Oliveira, 2011; McInnis, Strand e Webb, 2012). O conceito foi proposto por Hepler e Strand (1990) e desenvolvido como prática profissional por Cipolle, Strand e Morley (2012) para atender à demanda de redução da morbimortalidade relacionada ao uso de medicamentos. A filosofia da prática orienta atitudes e valores para que o farmacêutico assuma a responsabilidade pelas necessidades farmacoterapêuticas do paciente de forma holística e centrada na pessoa. O papel principal desse profissional ao cuidar de um paciente é a identificação, prevenção e resolução de problemas relacionados ao uso de medicamentos (PRM). Para isso, o profissional avalia se todos os medicamentos utilizados pelo paciente são indicados, efetivos e seguros, para então fomentar sua adesão aos respectivos produtos. Ao seguir essas etapas, o farmacêutico determina se o paciente apresenta algum PRM, elabora planos de cuidado para solucioná-lo em colaboração com o paciente e equipe de saúde e, posteriormente, avalia os resultados da sua intervenção (Oliveira, 2011; Cipolle, Strand e Morley, 2012).

Diversos trabalhos evidenciam os impactos positivos dessa prática, tanto para o paciente quanto para os profissionais envolvidos no cuidado, e ainda os benefícios associados à inserção desse serviço clínico nas equipes multidisciplinares (Isetts et al., 2008; Oliveira, Brummel e Miller, 2010; Mourão et al., 2013; Hirsch et al., 2014; Obreli-Neto et al., 2015; Mendonça et al., 2016; Detoni et al., 2017; Oliveira et al., 2017).

Por sua vez, diversos outros trabalhos discutem os desafios encontrados na implementação de serviços clínicos baseados em atenção farmacêutica em todo o mundo (Oliveira et al., 2005; Roberts et al., 2005; Dugan, 2006; Uema et al., 2008; Elrod et al., 2012; Sir et al., 2014). Todos eles apontam como dificultadores a formação tradicional do farmacêutico e a ausência de percepção da necessidade do cuidado farmacêutico pelos próprios farmacêuticos, por outros profissionais de saúde e pacientes. No Brasil, a formação tradicional do farmacêutico não é voltada para o cuidado em saúde; assim, não instrumentaliza os profissionais com as competências necessárias para a prática clínica nem os orienta para a tomada de decisão racional em farmacoterapia (Mendonça, Freitas e Oliveira, 2017). Essa formação ainda privilegia as ciências farmacêuticas e os conhecimentos e habilidades técnicos e tecnológicos, voltados ao produto, o medicamento (Oliveira, 2011, 2012). Em consequência, farmacêuticos têm se sentido inseguros em relação à oferta do cuidado como membros dos núcleos de apoio à saúde da família (NASFs), equipes de saúde multiprofissionais que oferecem apoio especializado às equipes de saúde da família (eSF) na atenção primária à saúde (APS) (Brasil, 1990, 2008, 
2013; Nakamura e Leite, 2014, 2016). As eSF, coordenadoras do cuidado nas redes de atenção à saúde do Sistema Único de Saúde (SUS), acionam a equipe multiprofissional sempre que identificam a demanda de suporte de outro profissional de saúde.

Diversos profissionais podem constituir o NASF. Cada município elege a composição dessa equipe de acordo com o diagnóstico de suas necessidades territoriais. A proposta de trabalho para os farmacêuticos no NASF prevê a divisão de sua carga horária (normalmente de quarenta horas semanais) entre atividades técnico-gerenciais (gestão das farmácias das unidades de saúde) e técnico-assistenciais (atividades diretas com pacientes e equipe de saúde, como visitas domiciliares, atendimentos individuais ou compartilhados com outros profissionais de saúde, atividades coletivas e apoio técnico-pedagógico às eSF). De acordo com as diretrizes do NASF, é importante que seja desenvolvido acompanhamento farmacoterapêutico para pacientes com regime terapêutico complexo (Brasil, 2009).

Nesse contexto, acredita-se que a prática profissional da atenção farmacêutica, ofertada como serviço clínico, seja adequada para a realidade do país, destacando-se a importância da utilização de um método de tomada de decisão racional sobre farmacoterapia pelo farmacêutico a fim de otimizar o uso de medicamentos pelos usuários do SUS (Angonesi e Sevalho, 2010; Oliveira, 2011).

Ao considerar o contexto apresentado, o estudo que originou este artigo teve como objetivo compreender os elementos essenciais do processo de sistematização da prática clínica farmacêutica no NASF, com base no referencial teórico-metodológico da atenção farmacêutica. Almeja-se que os resultados contribuam para que as instituições de saúde e os próprios farmacêuticos visualizem um possível caminho a trilhar rumo ao cuidado e à oferta de serviços de GTM.

\section{Metodologia}

O estudo foi desenvolvido em NASFs vinculados às unidades de atenção primária à saúde (UAPSs) de Belo Horizonte, Minas Gerais, Brasil. Seis farmacêuticos de equipes do NASF do referido município participaram do estudo, todos com formação tradicional em Farmácia, porém com conhecimento do arcabouço teórico da atenção farmacêutica, e um deles em processo de sistematização da prática clínica com base nesse referencial. De acordo com os Cadernos de Atenção Básica, que norteiam o trabalho do NASF (Brasil, 2009; 2014), os farmacêuticos devem dividir sua carga horária entre dois grandes eixos, atividades técnico-gerenciais e assistenciais. É recomendado que essa divisão seja paritária, mas orienta-se que o profissional seja capaz de administrá-la a fim de atender de forma adequada à demanda do serviço de saúde do qual faz parte. Todas as atividades assistenciais desenvolvidas pelo 
NASF são oriundas de demandas identificadas pelas eSF. O principal espaço utilizado para essa discussão são reuniões entre eSF e NASF com periodicidade determinada em cada serviço, normalmente mensal, para que os casos sejam então matriciados ou discutidos. É nesse momento que os atores são identificados e envolvidos na condução/contribuição para o caso, devendo o plano de cuidado ser construído de forma compartilhada. Portanto, para que o farmacêutico possa se envolver e contribuir ativamente na construção desse plano de cuidado, esse profissional deve ser capaz de dominar seu núcleo de saber no âmbito clínico, compartilhar ações de cuidado na mesma ótica dos outros profissionais de saúde e organizar sua agenda para atender às múltiplas demandas que o cargo exige. Em relação aos farmacêuticos sujeitos do estudo, houve uma discrepância no tempo dedicado a cada tarefa. Normalmente essa divisão está hoje relacionada à demanda técnico-gerencial das farmácias da UAPS aliada ao perfil do profissional, que não é, tradicionalmente, clínico. A primeira autora do artigo é uma das farmacêuticas participantes da pesquisa e dedica em média vinte horas por semana para atividades relacionadas à prática clínica, carga horária dividida de forma paritária entre atendimentos individuais, visitas domiciliares, grupos operativos e participação em reuniões de matriciamento.

Foi realizada pesquisa qualitativa autoetnográfica (Jones, 2005; Anderson, 2006; Ellis, Adams e Bochner, 2011). Os métodos para coleta de dados foram: observação participante no período de outubro de 2014 a outubro de 2015, com utilização de diário de campo para registro de situações vivenciadas e escrita reflexiva sobre o processo de sistematização da prática clínica farmacêutica no NASF (Mulhall, 2003; Sandberg, 2005). Realizaram-se entrevistas semiestruturadas com os farmacêuticos participantes, utilizando-se tópico-guia, com o objetivo de elucidar e validar as percepções da pesquisadora autora principal na fase de observação participante (Chang, Ngunjiri e Hernandez, 2013). O processo de coleta de dados foi interrompido segundo o critério de saturação (Creswell, 2013). Todos os dados gerados foram analisados com o auxílio do software NVivol0, a fim de identificar os elementos essenciais do fenômeno sob investigação (Minayo, 2010).

Esta pesquisa obedece à resolução n. 466/2012 e foi aprovada pelo Comitê de Ética em Pesquisa da Universidade Federal de Minas Gerais (UFMG) e da Secretaria Municipal de Saúde de Belo Horizonte, com Certificado de Apresentação para Apreciação Ética n. 25780314.4.3001.5140.

\section{Resultados e discussão}

Nos resultados, procurou-se discutir as reflexões autoetnográficas, com incorporação das experiências de outros participantes sobre o processo de sistematização de uma prática clínica farmacêutica na APS. O tema central é 
'proposta de integração de um serviço de GTM nos fluxos das unidades de saúde' e está exposto no final, após se passar por outros dois temas conectados a ele que emergiram ao longo da análise de dados: 'construção de uma nova identidade profissional na equipe multidisciplinar' e 'incorporação de novas atividades na rotina de trabalho'. Cada tema apresenta subcategorias, conforme apresentado a seguir:

\section{Construção de uma nova identidade profissional na equipe multiprofissional}

Trabalhar de forma multiprofissional tem sido um desafio, pois é uma estratégia relativamente recente para o farmacêutico, considerando sua inclusão de forma sistemática na APS a partir de 2009. Essa abordagem tem a proposta de desenvolver uma cultura de compartilhamento de saberes e relacionamento horizontal e igualitário entre os profissionais (Campos e Domitti, 2007). Tal é o enfoque do apoio matricial do NASF e se materializa de forma pactuada nas intervenções, levando em conta a clareza das responsabilizações comuns e específicas de cada profissional envolvido, das eSF e do NASF. Deve produzir um cuidado continuado e longitudinal, próximo da população e na perspectiva da integralidade (Brasil, 2011).

O desafio do trabalho multiprofissional para o farmacêutico, portanto, é ter clareza da sua responsabilidade específica ao atuar em uma equipe de saúde, para então poder fazer parte do compartilhamento de saberes e das responsabilizações comuns (Nakamura e Leite, 2014).

Discutimos três subcategorias dentro desse tema: 'o farmacêutico e a apropriação do GTM e de seu papel na equipe de saúde'; 'superação da invisibilidade do farmacêutico como profissional potencial para solucionar problemas clínicos e propor intervenções por meio do plano de cuidado em prontuário'; e 'construção de parcerias'.

\section{O farmacêutico e a apropriação do GTM e de seu papel na equipe de saúde}

À medida que o farmacêutico se familiariza com o papel correspondente ao de um profissional de saúde, ele passa a compreender até onde deve ir o seu cuidado e como deve realizar referenciamentos de forma adequada. Além disso, passa a dedicar mais tempo na sua função de atendimento individualizado, avaliando indicação, efetividade, segurança e conveniência do uso dos medicamentos de seus pacientes em vez de ser um profissional que não é percebido como necessário na equipe de saúde (Saar e Trevizan, 2007), ou simples fiscalizador de outras práticas, conforme exemplificado no trecho de diário de campo a seguir: 
Descobri que muito do que faço nos atendimentos/visitas não é da minha alçada, passo muito tempo perguntando se [o paciente] usa andador, se tem tapete no chão, se fica sempre na mesma posição etc. Minhas condutas estão incompletas e equivocadas, o único padrão que tenho, ou tinha, é trabalhar adesão em todos os atendimentos, custe o que custar. Quando me impeço de fazer isso, fico sem saber o que fazer ali (trecho de diário de campo).

Com a apropriação de sua prática profissional, o farmacêutico começa a compartilhar suas percepções com sua equipe de trabalho e pode-se perceber uma abordagem mais centrada no paciente para a utilização de medicamentos. A equipe passa a compreender que existem outros 'problemas' além da adesão ao medicamento:

Hoje a nutricionista fez uma consideração ótima! Disse que ia pedir uma visita [com o farmacêutico] para o paciente que ela atendeu hoje, pelo fato dele não usar um dos medicamentos de hipertensão porque sente tontura. Ela lembrou que eu tinha dito que a dose pode estar alta e falou: 'Será que é isso? Vou agendar uma avaliação sua' (trecho de diário de campo).

\section{Superação da invisibilidade do farmacêutico}

É comum a discussão de um caso clínico entre as diversas categorias profissionais em que claramente há necessidade de intervenção do cuidado farmacêutico, mas a equipe nem sempre tem essa percepção. $O$ farmacêutico usualmente não é incluído quando o paciente está com um quadro de saúde descompensado. O profissional que encaminha o paciente, em geral, não espera que o farmacêutico o auxilie na condução do caso ou no alcance de metas terapêuticas. O envolvimento desse profissional é mais comum quando se percebem problemas de adesão ao tratamento.

Além do despreparo do farmacêutico para se inserir no processo de cuidado, a complexidade inerente a esses casos traz a sensação de impotência e pode desestimulá-lo a se qualificar, pois ele compreende que os casos estão além da sua capacidade de cuidado. Portanto, o mais comum tem sido o farmacêutico ser acionado quando o paciente é um idoso com uma condição clínica que altere sua percepção do mundo (por exemplo: demências relacionadas ou não ao Alzheimer), que está sem cuidador, ou um idoso analfabeto com função cognitiva em declínio, conforme podemos observar na fala de uma entrevistada:

O paciente não adere, o paciente não quer tomar, e aí você vai lá fazer a 'mágica' para o paciente entender que ele tem que tomar. Será que esse é mais um dos 
famosos 'casos sem solução' que os outros farmacêuticos tanto falam? Eles dizem que têm a impressão que são encaminhados para a gente apenas os casos sem solução (Farmacêutico 1).

O profissional farmacêutico na APS claramente observa que os pacientes considerados 'rebeldes' com sua farmacoterapia são os referenciados para seu atendimento. Normalmente são pacientes com os quais as equipes já 'tentaram de tudo' e encaminharam para o farmacêutico como uma 'última tentativa'. Por ser um paciente considerado de 'última chance', ocorre com frequência de as equipes não se interessarem mais pelo caso, devido ao desgaste natural da relação terapêutica, ocorrido nas diversas tentativas anteriores de controle dos problemas de saúde.

Eles começaram o matriciamento assim: não sei se esse caso é pra você, Dani, mas a gente tá sem saber o que fazer com ele, na verdade é só pra dizer que não tentamos (trecho de diário de campo).

Nesse contexto, é importante que o farmacêutico auxilie os outros profissionais de saúde a compreenderem o seu papel de colaboradores para a estabilização de quadros de saúde, indo além de suas atribuições na garantia da adesão aos medicamentos, como exemplificado na fala a seguir:

Ela (ACS) até o havia convencido (com muito trabalho, ela frisou) a me aguardar, mas que ela mesma não via muita necessidade da visita, uma vez que os medicamentos estão organizados (trecho de diário de campo).

É importante ainda apontar a invisibilidade de muitas das avaliações e propostas do plano de cuidado farmacêutico no âmbito da atenção primária e da secundária. Apesar de haver um sistema de registro em comum, não é esperado que haja avaliação de condutas estabelecidas por um farmacêutico antes de uma especialidade médica fazer suas intervenções.

A implantação dos planos de cuidado tem sido um desafio. Os médicos, em geral, não leem a evolução [do farmacêutico], principalmente em se tratando dos especialistas. É um fato. Agora ficam esses PRM pendentes toda a vida, e o paciente só piorando (trecho de diário de campo).

Tais situações são causas de muitas frustrações tanto para o profissional quanto para o paciente, pois pode haver uma descontinuação do cuidado pela impossibilidade de resolver os PRMs identificados. Apesar de haver um sistema de referência e contrarreferência, ele é sabidamente falho. Mecanismos de 
superação dessa dificuldade devem ser discutidos entre os diferentes níveis de atenção (Campos e Domitti, 2007; Mendes, 2012).

Falhas de comunicação representam importantes desafios do trabalho em equipe multidisciplinar e de apoio matricial. Sugerem-se assim modificações entre as relações dos níveis hierárquicos em sistemas de saúde que, por sua vez, apresentam obstáculos estruturais, éticos, políticos, culturais, epistemológicos e subjetivos ao desenvolvimento desse tipo de trabalho integrado em saúde (Campos e Domitti, 2007). Para poder participar da construção e da superação desses obstáculos, o profissional farmacêutico deve se envolver diretamente no cuidado de pacientes, e assim gradativamente ganhará reconhecimento por sua atuação profissional assistencial pelo restante dos integrantes de uma equipe de saúde (Sorensen et al., 2016). Ademais, a construção da colaboração interprofissional precisa ser estabelecida além da atenção primária.

É importante ainda o estabelecimento de critérios de encaminhamento em que o profissional possa ser aproveitado sem ser a 'última alternativa' para aquele paciente, de modo a auxiliar na construção do cuidado ao mesmo tempo que os outros profissionais desenvolvem o seu núcleo de saber. Além disso, é necessário haver uma conscientização de que se o paciente foi encaminhado para mais um profissional - no caso, o farmacêutico -, a equipe deve estar ciente e preparada para dar suas contribuições nas avaliações que se fizerem necessárias. Portanto, cabe à equipe a ciência de que essa é mais uma tentativa, de todos em conjunto, não apenas do farmacêutico. Campos e Domitti (2007) observam que alguém deve se responsabilizar pelo seguimento longitudinal e pela construção de uma lógica que procure integrar a contribuição dos vários serviços, departamentos e profissionais. Em geral, esse papel cabe aos integrantes da equipe de referência, mas como enfatizam os autores citados, essa não é a tradição de funcionamento dos serviços de saúde.

\section{Construção de parcerias}

Um bom relacionamento com todos da equipe é de primordial importância para qualquer equipe multiprofissional. Entretanto, para o trabalho clínico do farmacêutico, as parcerias se estreitam, principalmente com os prescritores. Ela é fundamental, pois uma visão profissional pode vir a ser o complemento da outra (Giberson, Yoder e Lee, 2011; Cipolle, Strand e Morley, 2012; Elrod et al., 2012; Sorensen et al., 2016). Um profissional aprende com o outro, conforme o exemplo a seguir.

As condutas assertivas do GTM podem estimular o profissional médico a dar continuidade na avaliação clínica adequada do paciente, que não estava sendo feita (trecho de diário de campo). 
A familiarização dos prescritores com o cuidado provido pelo farmacêutico é essencial para evitar julgamentos inadequados da função específica de cada profissional. Quando ambas as partes estão bem esclarecidas e os papéis bem definidos, a tendência é uma troca de saberes com apoio mútuo, construção de uma relação profissional pautada na corresponsabilização e na busca de melhores alternativas de abordagem ou tratamento para o paciente que transita fora das metas terapêuticas.

Quando perguntei se os pacientes tinham exames recentes, não entenderam. Por que eu precisaria saber o estado de saúde do paciente? (trecho de diário de campo).

Do contrário, quando o profissional médico não está ciente das condutas do farmacêutico, naturalmente advindas de uma abordagem que pretende atender a todas as necessidades farmacoterapêuticas do paciente (Oliveira, 2011 ; Cipolle, Strand e Morley, 2012), pode haver conflitos e desgaste do relacionamento profissional, com impacto na relação terapêutica farmacêuticopaciente - pois condutas não acordadas são ignoradas, principalmente aquelas que dependem de avaliação médica, como a fala a seguir representa:

Outra tipificação diz respeito ao relacionamento entre o farmacêutico que está tentando ser provedor de GTM e outros profissionais de saúde. As seguintes situações podem representar um mistério: por que preciso de exames? Por que estou sugerindo a alteração na dosagem de medicamentos ao invés de treinar o paciente a usá-los? Por que estou atendendo pacientes em vez de estar na farmácia? As condutas realmente se alteram quando o outro profissional tem um 'face a face' com o meu 'novo' trabalho (trecho de diário de campo).

O enfermeiro é parceiro importante e pode ser exemplo na forma como tem conquistado autonomia e destaque em seu processo de cuidado. Ele é, muitas vezes, peça-chave para a coesão da eSF e ponto de apoio para a coordenação do cuidado. Deve ser visto como um importante ponto de auxílio aos encaminhamentos e demandas oriundos do oferecimento do serviço de GTM, conforme ilustrado pela fala a seguir:

Fiz um plano de cuidado, agora é só acionar a enfermeira para auxílio nos encaminhamentos. Se não fosse o GTM, eu trabalharia somente adesão. Ponto. E nem identifiquei adesão como um problema (ainda). E ele [paciente] poderia sequelar e eu nem desconfiaria. Encontrei a enfermeira e mencionei a finalização. Ela se prontificou a olhar porque é um caso grave e ela ia sair de férias. Falou que liga na geriatria se necessário for pra dar a contrarreferência. Fiquei aliviada (trecho de diário de campo). 
Ao se considerar o fluxo de discussão de casos do NASF, há um espaço de ocorrência periódica destinado à problematização, ao planejamento, à programação e à execução de ações colaborativas entre NASF e equipes de saúde, o matriciamento. Este engloba discussões de casos e temas e deve pautar-se em uma relação dialógica e horizontal entre os profissionais que compõem tais equipes, além de baseada em uma abordagem centrada na pessoa e com enfoque intersubjetivo e interdisciplinar (Brasil, 2014). Entretanto, o matriciamento não é apenas uma reunião, mas um momento de troca de saberes em que os profissionais ampliam seu escopo de ação e encontram a interdisciplinaridade em sua prática cotidiana, em qualquer encontro com essa finalidade. O trecho a seguir, de um entrevistado, exemplifica:

Ultimamente eu tenho procurado direto o profissional para casos que não podemos aguardar o matriciamento. Porque a reunião do NASF demora trinta dias para você ter um retorno. Aí você negocia aquilo com o paciente, para poder tentar implantar essa intervenção. Aí você vai esperar mais outro mês se acabou de ter uma reunião para poder fazer isso? Então acho que acaba que frustra a pessoa e coloca o paciente em risco (Farmacêutico 2)

Dessa forma, é possível que, de acordo com a avaliação inicial e as necessidades do paciente identificadas pelo farmacêutico, ele observe a necessidade de se dirigir à equipe de referência antes da data da reunião de matriciamento para acordos de ações e intervenções. Em situações que exijam atenção específica ao núcleo de saber do apoiador, este pode programar para si mesmo uma série de atendimentos ou de intervenções especializadas. Para isso, deve manter contato com a equipe de referência, que não deve se descomprometer com o caso. Ao contrário, deve procurar redefinir um padrão de seguimento complementar e compatível com o cuidado oferecido pelo apoiador diretamente ao paciente, à família ou à comunidade (Campos e Domitti, 2007).

O momento do matriciamento promove, aos poucos, o contorno das questões que envolvem a invisibilidade do profissional farmacêutico, por meio da construção de parcerias, conforme observam Sorensen e colaboradores (2016). Promove ainda encontro entre distintas perspectivas, convidando os profissionais a comporem projetos terapêuticos que incluam outras racionalidades e visões de mundo. Em casos de impasse, e se o impasse se referir ao esquema terapêutico, não há instâncias superiores para resolver o conflito. A referência da equipe e condutora do caso, em geral, tem pequena capacidade de interferir sobre a conduta específica do especialista, ao se tratar do apoiador matricial, cabendo aos envolvidos no conflito encontrar uma saída que não prejudique o usuário nem paralise o projeto terapêutico (Campos e Domitti, 2007). 


\section{Incorporação de novas atividades na rotina de trabalho}

O trabalho em saúde tem características próprias que incluem um conjunto de saberes e práticas com a finalidade de realizar uma intervenção sobre determinado problema de saúde, conforme os critérios adotados pelo modelo de atenção do serviço, ao considerá-lo como um objeto de ação de saúde (Bueno e Queiroz, 2006). Em se tratando do cuidado farmacêutico, o objeto de cuidado é a pessoa, com os problemas relacionados ao uso de medicamentos, objetivos e subjetivos, que ela possa estar vivenciando (Oliveira, 2011 ; Cipolle, Strand e Morley, 2012).

Além disso, é necessário que o profissional seja capaz de transitar entre os diversos pontos do cuidado com segurança e entendimento do seu papel, para estruturar uma agenda que seja capaz de cumprir com os requisitos mínimos de suas atribuições (Brasil, 2009; Nakamura e Leite, 2014, 2016). Nakamura e Leite (2016) observaram que o farmacêutico, por não ter uma formação em saúde que privilegia habilidades para a tomada de decisão clínica, é um profissional limitado para participar de um compartilhamento de casos de forma equânime, quando sua função está além da atividade 'esperada' relacionada à gestão do medicamento.

O processo de autonomia pressupõe que o profissional possa interferir no processo de definição das prioridades na assistência, de acordo com seu núcleo de saber. Está embasado na direção da vontade do indivíduo para a ação, mediante influências sociais e culturais, e deve ser construído juntamente com atitude profissional (Bueno e Queiroz, 2006). Em que pese a autonomia profissional, discutimos os tópicos relacionados à incorporação de novas atividades na rotina do profissional farmacêutico no NASF: solicitação e interpretação de exames, documentação da prática de GTM e prescrição farmacêutica.

\section{Solicitação e interpretação de exames}

Para embasar suas decisões com segurança e dar seguimento ao processo de cuidado, o profissional farmacêutico lança mão de elementos, como qualquer outro profissional de saúde (por exemplo: exames laboratoriais). A fala de uma entrevistada exemplifica a questão:

Sinto muito essa fragmentação, essa falta de autonomia, de entendimento e diálogo dos profissionais. 'Por que você está querendo a hemoglobina glicada desse paciente?', me perguntam. 'O que ela tem a ver com a tuberculose?' (Farmacêutico 4).

O objetivo do trabalho clínico do farmacêutico, ou do serviço de GTM, é avaliar indicação, efetividade, segurança da farmacoterapia e, ainda, realizar encaminhamentos para fins diagnósticos, quando necessário. Assim, para 
efetivar o processo de avaliação da farmacoterapia do paciente, o farmacêutico precisa avaliar parâmetros de efetividade e segurança que, na maioria das vezes, são fornecidos por exames laboratoriais. Entretanto, no local estudado, esse profissional não tem autonomia para solicitar tais exames. Apesar de o NASF ser uma retaguarda especializada e copartícipe do cuidado, cada profissional deve acionar a eSF quando necessário para apoiar suas decisões ou discutir pontos estratégicos e compartilhados de intervenção. Ao considerar que exames são apenas um dos respaldos para a tomada de decisão clínica do farmacêutico, ele se vê obrigado a acionar a eSF antes de efetivamente ter elementos para a discussão do caso.

Uma abordagem menos dependente da eSF e que usa de racionalidade para acioná-la (por exemplo: discussão das intervenções que porventura precisem de avaliação médica, envolvimento de outros profissionais) pode ser uma estratégia interessante para aumentar a eficiência do trabalho das equipes de saúde, de modo a reduzir a sobrecarga de trabalho de cada profissional e agilizar o processo de cuidado. Existem experiências no Brasil que apontam que o serviço de GTM pode ser estruturado na APS desde que a autonomia de trabalho seja garantida ao profissional (Mourão et al., 2013; Obreli-Neto et al., 2015; Silva et al., 2016). Além disso, foi necessária a construção de novas perspectivas para atuação do farmacêutico provedor do cuidado em GTM em relação aos outros profissionais de saúde (Oliveira et al., 2015). Entretanto, deve-se ter o cuidado de não promover a já estabelecida supervalorização da autonomia profissional, de modo a julgar o profissional ser capaz de deliberar sobre casos de modo isolado e definitivo, desconsiderando a ação interdisciplinar característica do trabalho na APS (Campos e Domitti, 2007).

\section{Documentação da prática}

A documentação sistematizada gera base indispensável para a construção de um processo de cuidado focado na responsabilização profissional e na produção de resultados. É só por meio dela que se percebe o quanto se pode fazer pelo paciente e qual o melhor caminho para fazê-lo. O uso de um prontuário eletrônico que permita o desencadear do raciocínio lógico e a tomada de decisão em GTM tem favorecido o processo de aprendizado do profissional e aumentado a eficiência do processo, como exemplifica a fala a seguir:

Descubro a cada nova evolução que eu deixei várias lacunas, quando lanço no prontuário eletrônico. Ele me ajuda a me acostumar com o raciocínio e favorece a tomada de decisão, porque fica tudo muito mais lógico e claro (trecho de diário de campo). 
Ademais, a documentação sistematizada permite que apenas os pontos de intervenção sejam compartilhados no prontuário único do serviço em que o profissional está inserido, de modo a fortalecer o papel do profissional e evitar a invisibilidade de suas ações no cuidado.

Entretanto, é nesse momento que o profissional tem a percepção do volume de informações a serem recuperadas do prontuário médico do paciente para serem combinadas com as informações colhidas na avaliação inicial do farmacêutico, complementando-a. E então ele passa a encarar essa retomada como um desafio para ser efetivo em sua prática, pois o fator tempo parece não favorecer a qualidade da coleta de dados e, consequentemente, das tomadas de decisão, principalmente do profissional em processo de aprendizado.

Por não existir no Brasil uma diretriz que norteie as prioridades de encaminhamento para um provedor de GTM na APS, é comum os pacientes encaminhados terem mais de seis condições de saúde (dados de registros pessoais) sendo tratadas simultaneamente e com um grande número de medicamentos associados. Ao se considerarem a novidade do serviço e o processo de aprendizado vivenciado pelo farmacêutico, isso pode trazer uma grande carga de angústia para o profissional no dia a dia. O volume de informações a serem registradas é proporcional às comorbidades associadas a esse paciente. Atualmente, por questões diversas, nem sempre os profissionais do NASF contam com um local adequado que possa proporcionar o atendimento e a evolução sequencial, sendo necessária a criação de estratégias que favoreçam os registros. O NASF, assim como outras equipes multidisciplinares que apoiam as equipes de saúde na APS, ainda está em fase de superação de diversas barreiras - culturais, organizacionais e estruturais, entre outras (Campos e Domitti, 2007). A estruturação da agenda com proteção do horário de evolução foi a saída encontrada para a superação do não registro, uma situação que deve ser considerada grave e impactante para o desenvolvimento do processo de cuidado.

Então protegi minha agenda todas as sextas de manhã para evolução de pacientes e observei que a organizando com atendimentos apenas segundas e quartas tenho conseguido ficar com o tempo organizado para a farmácia e evoluções (trecho de diário de campo).

Além da proteção do momento de registro, o equilíbrio entre novos casos e retornos otimiza significativamente a agenda. O profissional deve ser capaz de se organizar para disponibilizar a agenda para casos novos - que demandam mais tempo tanto para o atendimento propriamente dito quanto para o registro - e para os retornos, normalmente mais rápidos, com o objetivo de verificação de orientações dadas anteriormente e que contam com um 
registro já em andamento, sendo necessário apenas atualizá-lo. Quanto mais completos e bem feitos a primeira avaliação e o registro, mais simplificada se torna a conduta do caso e a agilidade do profissional nos retornos - o que é exemplificado na fala a seguir:

Fiz um retorno seguindo minha própria evolução anterior, foi ótimo, os resultados dos exames não haviam saído ainda, mas já fui escrevendo as considerações em cada condição de saúde. Depois só atualizar com os resultados e pronto. Esse foi rápido e não me senti perdida (trecho de diário de campo).

A estratégia de registro prévio dos exames disponíveis no sistema tem trazido resultados positivos ao favorecer o processo de tomada de decisão.

Quando possível, anoto os exames dos pacientes do dia ou da semana, isso me ajuda a decidir na hora do atendimento o quão efetivo e segura é a farmacoterapia para fomentar a adesão, se necessário for. Achei que agiliza em muito o processo. Se o paciente não tem exames recentes, eu já posso providenciar a solicitação (trecho de diário de campo).

Sorensen e colaboradores (2016) apontam o suporte ao processo do cuidado farmacêutico como fundamental para o sucesso dos serviços de GTM - o que inclui ferramentas administrativas, tais como acordos de colaboração com outros profissionais para a construção de parcerias, abordados anteriormente, normas de documentação e o processo de encaminhamentos (referência e contrarreferência). Além disso, esses autores enfatizam que um elemento crítico para um programa bem-sucedido é a capacidade de se compartilharem recursos em grandes organizações, bem como ter um modelo de prática consistente, ou padrões de prática, para serviços de GTM que devem ser seguidos por todos os provedores farmacêuticos. É importante que todos falem a mesma língua, de modo a uniformizar condutas e favorecer o reconhecimento do profissional e a avaliação do impacto do seu trabalho na saúde dos pacientes.

\section{Prescrição farmacêutica}

$\mathrm{O}$ ato de prescrever não se restringe a medicamentos; está relacionado também a terapias não farmacológicas que o profissional prescreve, responsabilizando-se por aquela orientação. Todo o processo prescritivo deve ser registrado (Conselho Federal de Farmácia, 2013).

Além disso, ao serem descritas as etapas do processo da prescrição, essa atividade/atribuição é posicionada como resultado de um processo de cuidado, semelhante ao dos demais profissionais de saúde. Entende-se que a prescrição 
farmacêutica só poderá ser realizada mediante consulta e exige a avaliação dos resultados advindos das intervenções propostas no atendimento. Assim, o ato de prescrever correlaciona-se ao ato de cuidar e, necessariamente, envolverá uma decisão clínica para definição das condutas, seja para prescrever medicamentos associados a protocolos clínicos previamente acordados no sistema de saúde em questão, ou medicamentos isentos de prescrição, seja para fornecer orientações não farmacológicas. Conforme demonstrado, o processo da prescrição apresenta características que podem ser respaldadas pelo arcabouço teórico-metodológico do serviço de GTM. Entretanto, vale ressaltar que o ato prescritivo do farmacêutico não é alvo desse serviço.

A formação adequada do profissional farmacêutico para avaliar e identificar problemas e recomendar (prescrever) intervenções para resolvê-los pode ir ao encontro de uma prática clínica farmacêutica resolutiva. O método empregado pelo GTM, além de delimitar a responsabilidade do farmacêutico clínico, racionaliza a busca de causalidade e organiza a solução de problemas, contribuindo para o desenvolvimento das competências necessárias ao processo prescritivo muito além do uso de medicamentos, conforme exemplificado na fala a seguir:

Quais são as condutas majoritárias que devem ser seguidas para uma atuação equilibrada de um profissional que faz GTM, farmacológicas, não farmacológicas? A finalidade é pensar no bem-estar do paciente em relação à sua farmacoterapia, e com certeza sistematizar as condutas por meio dos PRM, e não suprir uma falha do sistema ou de um profissional, e de repente envolvermos com a prescrição de forma tão intrínseca que não conseguiremos olhar [o paciente] de forma holística. O farmacêutico tem dificuldade de se posicionar, a reflexão e responsabilização da prática de GTM favorece a responsabilização do ato prescritivo, de qualquer orientação a ser dada (trecho de diário de campo).

Em se tratando do farmacêutico do NASF, como o GTM organiza o processo de cuidado farmacêutico e é, necessariamente, uma prática colaborativa, a demanda pela prescrição pode surgir 'naturalmente' entre farmacêutico e médico. Contudo, ainda é uma construção que exige tempo e o fortalecimento dos vínculos entre profissional e eSF, além de clareza da responsabilização e competência clínica do farmacêutico para tal atividade. A possibilidade de prescrever favorece a autonomia profissional e reduz o tempo de espera para a solução de problemas, com benefício direto para o paciente e indireto para o médico, que pode ter sua demanda relativamente reduzida e passar mais tempo com pacientes que demandem avaliações médicas mais detalhadas.

Para garantir a autonomia necessária para ser gestor de sua prática e compartilhar saberes, que é um reflexo da segurança e da familiaridade de sua prática clínica, o farmacêutico, como qualquer outro profissional de saúde, 
deve fazer um esforço constante e perene na aquisição ou atualização do conhecimento necessário para exercício da sua prática profissional (Silva et al., 2016). E como exposto, o GTM pode nortear essa transformação.

\section{Integração de um serviço de GTM nos fluxos da unidade de saúde}

Entende-se que o trabalho multiprofissional exige negociação, diálogo contínuo, postura ética e corresponsabilização por parte de todos os envolvidos. Nesse sentido, é fundamental que todos os participantes da equipe compreendam a nova identidade do farmacêutico como cuidador com corresponsabilidade sobre os resultados da farmacoterapia do paciente.

Ao se tratar de coparticipação e corresponsabilização, não é o 'fazer junto o tempo todo' nem o 'centralizar tudo na reunião de matriciamento' que garantirá tal cenário. É importante que os profissionais compreendam o conjunto de necessidades dos usuários e estejam dispostos a compartilhar o cuidado, nos diferentes modos em que isso pode ocorrer (Brasil, 2009). Para tanto, abordemos as subcategorias 'qualificação dos encaminhamentos para o serviço de GTM' e 'qualificação da agenda e dos processos de trabalho do farmacêutico no NASF'.

\section{Qualificação dos encaminhamentos para o serviço de GTM}

Quando não se espera que o farmacêutico faça avaliações clínicas e exerça um papel de responsabilização pela farmacoterapia do paciente, é comum que os encaminhamentos dos pacientes ocorram em 'conversas de corredor' (Nakamura e Leite, 2016). Não há uma oficialidade de solicitação do cuidado farmacêutico. No início do processo de inserção do farmacêutico na equipe, é comum se lembrar de um paciente que não usa os medicamentos corretamente, ao final de uma reunião de matriciamento, e solicitar-se a intervenção do farmacêutico sem nenhum dado a mais, como indica a fala a seguir:

Os casos passados pra mim são no corredor, na reunião eles só levam a demanda do paciente. Será que porque pra mim a demanda é da equipe? Para a fono, a fisio, nutri, é comum o paciente que traz encaminhamento de outro nível de atenção, então se torna 'oficial'. E eu falo: por que vocês não passam o caso com os detalhes igual o dos outros na reunião? Eles riem e não respondem (trecho de diário de campo combinado à entrevista).

Com o passar do tempo e a superação parcial das questões apontadas acerca da invisibilidade do profissional farmacêutico, os casos tendem a ser mais bem direcionados e com situações que tangenciam o não alcance de me- 
tas terapêuticas, independentemente de preocupações com a adesão. A fala a seguir exemplifica esse processo:

Hoje no matriciamento tinha dois casos para mim! A novidade é que eles foram encaminhados pela médica! Ela até tinha levado quais os medicamentos que os pacientes usavam e quais as modificações que ela já havia feito na farmacoterapia devido a resultados de exames recentes, e queria que eu desse continuidade em outras questões (trecho de diário de campo).

Deve-se levar em consideração que pacientes referenciados, em detrimento dos que buscam ativamente o serviço, tendem a apresentar quadros de saúde mais complexos (Cipolle, Strand e Morley, 2012). Quanto mais bem qualificado o encaminhamento, maior a chance de as intervenções serem compartilhadas e ágeis. Se o paciente chega ao profissional com a demanda do cuidado e os elementos para o trabalho (por exemplo: exames recentes e disponibilidade real de suporte pela equipe), a probabilidade de o desfecho ser satisfatório é grande.

\section{Qualificação da agenda e processos de trabalho do farmacêutico no NASF}

A divisão do processo de trabalho farmacêutico em técnico-gerencial e técnico-assistencial parece favorecer uma grande carga de emoções negativas nos farmacêuticos. Esse item foi o assunto mais abordado em diário de campo e entrevistas. Ansiedade, angústia, esgotamento, interrupções constantes, preocupação, cansaço, sufocamento e frustração: alguns dos temas mais relevantes emergentes dos dados e que podem ser barreiras para o processo de sistematização da prática clínica desse profissional. Existem diversos outros estudos que confirmam essa percepção (Oliveira, 2003; Gastelurrutia et al., 2009; Sir et al., 2014). Os trechos de entrevista e diário de campo a seguir exemplificam:

Estou esgotadíssima. O prazer de ver o trabalho evoluir, as ideias se acertarem, as condutas padronizarem é muito grande, mas o simples afastamento da parte administrativa traz uma imensa sensação de angústia. $O$ farmacêutico que cuida da gestão da farmácia e do cuidado fica esquizofrênico. Às vezes eu penso: 'ah, se o paciente não vier, vai ser tão bom porque eu posso avaliar a farmácia nessa e nessa pendência'. Ou então termino um atendimento, mas nem sempre posso evoluir, porque a farmácia está com outra demanda, isso corta o meu raciocínio (trecho de diário de campo combinado a trecho de entrevista).

Deve-se considerar que a prática constante promove maior capacitação do profissional, que será capaz de agilizar seus atendimentos ao conseguir 
ser mais objetivo, sem abandonar a filosofia centrada no outro, e avaliar a farmacoterapia do paciente de forma compreensiva.

Otimizar a oferta do serviço de GTM de modo que ele possa beneficiar o maior número de pessoas possíveis, portanto, pode ser uma conquista histórica para o Brasil. Entretanto, inicialmente, deve-se ofertar esse cuidado priorizando o número de pacientes suficientes para garantir a qualidade do serviço, de forma a gerar indicadores que estimulem o desenvolvimento da prática. Os pontos estratégicos levantados na pesquisa aqui apresentada se assemelham aos do estudo de Sorensen e colaboradores (2016), os quais observaram que após o serviço de GTM estar bem estabelecido dentro do sistema de saúde, pôde-se então desenvolver estratégias para expandir e otimizar recursos. Uma estratégia é selecionar a população mais adequada para ser encaminhada ao serviço de GTM (Oliveira, 2011; Sorensen et al., 2016). É desejável que sejam construídas diretrizes do serviço para seleção do público-alvo a fim de orientar os profissionais da eSF, do NASF e outros farmacêuticos da instituição. Pacientes sem alteração do regime farmacoterapêutico há mais de um ano e que podem estar fora dos objetivos terapêuticos, pacientes usuários de mais de cinco medicamentos para controle de condições crônicas, com ou sem problemas relacionados à adesão (Secoli, 2010), são público-alvo em potencial. Enfim, pacientes que necessitam de adequação do regime terapêutico para alcançar a estabilização das condições crônicas de saúde podem ser potenciais elegíveis para o atendimento no serviço de GTM. O diagnóstico epidemiológico poderá auxiliar as equipes de saúde a elegerem o público que mais se beneficiaria das ações do serviço.

A implementação do serviço de GTM no fluxo do NASF gerará indicadores que podem estimular a incorporação desse profissional como membro da eSF, conforme Mendes (2012) aponta. Na experiência do estudo aqui apresentado, considera-se que o serviço pode complementar de forma significativa o cuidado ao paciente, na ótica da resolutividade e da integralidade.

\section{Conclusão}

O estudo permitiu importantes ponderações sobre a cultura do farmacêutico e seu esforço de integração e exercício de uma prática clínica na APS. A escrita reflexiva estimulada pela autoetnografia trouxe à tona alguns meios para a construção da autonomia profissional, instigando a autora principal, em seu papel de participante do estudo, a compreender o espaço do cuidado e seus elementos constituintes. Ainda propôs reflexões acerca das modificações necessárias no cotidiano e no quadro coletivo para a integração do serviço de GTM como possível solução para a sistematização da prática clínica farmacêutica na comunidade. Para tanto, conforme o estudo apontou, é necessário 
que o próprio profissional seja transformado em sua maneira de agir e reflita em seu estar-no-mundo profissional.

O processo de pesquisa também foi capaz de auxiliar a identificação das bases necessárias para fomentar o processo de raciocínio e tomada de decisão em farmacoterapia pelo profissional farmacêutico. Habilidades clínicas aliadas ao fortalecimento de conhecimentos técnico-científicos e atitudes humanísticas têm se mostrado uma combinação adequada para a oferta do serviço de GTM com qualidade, enquadrando-se nas expectativas de uma APS resolutiva na perspectiva do cuidado integral e centrado na pessoa.

O estudo propôs ainda a discussão de pré-requisitos mínimos para se avançar no processo de integração desse serviço na APS: construção de uma nova identidade profissional na equipe multidisciplinar, incorporação de novas atividades e reorganização da rotina de trabalho. Em nível individual, apontou-se a necessidade de organização da agenda de trabalho do farmacêutico com priorização de tempo a ser dedicado a cada atividade, incluindo o tempo específico para dedicar ao cuidado e ferramentas que auxiliem nesse processo. Obter equilíbrio entre casos novos e retornos para que o profissional adquira experiência e desenvolva competência no cuidado foi uma alternativa promissora para o aprendizado na prática.

A união entre farmacêuticos para apoio na prática, com elaboração de documentos que possam agilizar e direcionar o processo e o estabelecimento de encontros periódicos para discussão de casos e trocas de impressões, pode ser movimento agregador do cuidado e impactante no esforço de sistematização da prática clínica farmacêutica. É necessário que o nível coletivo no qual o profissional esteja inserido seja partícipe desse interesse para que se favoreça o reconhecimento do papel do farmacêutico na equipe de saúde.

Para que o farmacêutico possa legitimar o seu trabalho no espaço do cuidado, é preciso mudar, transformar, reorganizar e reconstruir a sua prática. O arcabouço teórico da atenção farmacêutica, com sua filosofia, promove a mudança necessária, no sentido da soma com outros profissionais em busca do modelo transdisciplinar e biopsicossocial. A mudança cultural depende do esforço de cada um de nós. 


\section{Colaboradores}

Daniela Álvares Machado Silva foi responsável pela coleta e análise dos dados, além da redação do texto. Simone de Araújo Medina Mendonça, Djenane Ramalho de Oliveira e Clarice Chemello foram responsáveis pela orientação do trabalho e revisão crítica do manuscrito final.

Resumen Este estudio tuvo como objetivo comprender los elementos esenciales del proceso de sistematización de la práctica clínica del farmacéutico en la atención primaria de salud a partir del marco teórico de la atención farmacéutica, que subvenciona los servicios de gestión integral de la farmacoterapia. Auto etnografía fue la metodología cualitativa utilizada, construida en colaboración entre los autores. Los datos fueron producidos a través de observación participante, diarios de campo, reflexiones y entrevistas semiestructuradas con los farmacéuticos que desarrollan la práctica clínica en atención primaria de salud. Los resultados mostraron que los principales elementos para la sistematización de la práctica clínica son la ‘Construcción de una nueva identidad profesional en equipo multiprofesional' y la 'Incorporación de nuevas actividades en el trabajo de rutina' que, combinados, dan lugar a una 'propuesta de integración del servicio de gestión integral de la farmacoterapia en los flujos de las unidades de salud'. Por lo tanto, para que el farmacéutico pueda legitimar su papel en la atención al paciente, es necesario cambiar, transformar, reorganizar y reconstruir su práctica.

Palabras clave auto etnografia; atención primaria de salud; gestión de la farmacoterapia; atención farmacêutica.

\section{Notas}

${ }^{1}$ Universidade Federal de Minas Gerais, Belo Horizonte, Minas Gerais, Brasil.

$<$ dalvaresms@gmail.com>

Correspondência: Rua Radialista Ubaldo Ferreira, 17, apto. 1001, bloco 2, Castelo, CEP 31330-294, Belo Horizonte, MG, Brasil 


\footnotetext{
${ }^{2}$ Universidade Federal de Juiz de Fora, campus Governador Valadares, Minas Gerais, Brasil. simoneamm@gmail.com>

${ }^{3}$ Universidade Federal de Minas Gerais, Departamento de Farmácia Social, Belo Horizonte, Minas Gerais, Brasil.

$<$ djenane.oliveira@gmail.com>

${ }^{4}$ Universidade Federal de Minas Gerais, Departamento de Farmácia Social, Belo Horizonte, Minas Gerais, Brasil.

<clachemello@gmail.com>
}

\section{Referências}

ANDERSON, Leon. Analytic autoethnography. Journal of Contemporary Ethnography, v. 35, n. 4, p. 373-395, 2006.

ANGONESI, Daniela; SEVALHO, Gil. Atenção farmacêutica: fundamentação conceitual e crítica para um modelo brasileiro. Ciência \& Saúde Coletiva, Rio de Janeiro, v. 15, supl. 3, p. 3.603-3.614, 2010.

BRASIL. Lei n. 8.080, de 19 de setembro de 1990. Dispõe sobre as condiões para a promoção, proteção e recuperação da saúde, a organização e o funcionamento dos serviços correspondentes e dá outras providências. Diário Oficial [da] República Federativa da União, Poder Executivo. Brasília, DF, 20 set. 1990. Seção 1, p. 18.055.

BRASIL. Ministéro da Saúde. Portaria GM n. 154, de 24 de janeiro de 2008. Cria os Núcleos de Apoio à Saúde da Família - NASF. Diário Oficial [da] República Federativa da União, Brasília, DF, 25 jan. 2008. Seção 1, p. 47-49. Disponível em: <http://189.28.128.100/dab/ docs/legislacao/portaria154_24_01_08.pdf > . Acesso em: 16 abr. 2015.

BRASIL. Ministério da Saúde. Diretrizes do NASF: Núcleo de Apoio à Saúde da Família. Cadernos de Atenção Básica, Brasília, n. 27, 2010. 167 p. Disponível em: <http://189.28.128.100/ dab/docs/publicacoes/cadernos_ab/abcad27. pdf $>$. Acesso em: 25 out. 2017.

BRASIL. Ministério da Saúde. Portaria n. 2.488, de 21 de outubro de 2011. Aprova a Política Nacional de Atenção Básica, estabelecendo a revisão de diretrizes e normas para a organização da Atenção Básica, para a Estratégia Saúde da Família (ESF) e o Programa de Agentes Comunitários de Saúde (PACS). Diário Oficial [da] República Federativa do Brasil. Brasília, DF, 22 out. 2011. Disponível em: <http:// bvsms.saude.gov.br/bvs/saudelegis/gm/2011/ prt2488_21_10_2011.html>. Acesso em 25 out. 2017.

BRASIL. Ministério da Saúde. Política Nacional de Atenção Básica. Brasília, DF, 2012. Disponível em: <http://189.28.128.100/dab/docs/ publicacoes/geral/pnab.pdf $>$. Acesso em: 25 out. 2017.

BRASIL. Ministério da Saúde. Núcleo de Apoio à Saúde da Família: ferramentas para a gestão e para o trabalho cotidiano. v. 1. Brasília, $n$. 39, 2014. Disponível em: <http://bvsms.saude. gov.br/bvs/publicacoes/nucleo_apoio_saude_ familia_cab39.pdf $>$. Acesso em: 25 out. 2107.

BUENO, Flora M. G.; QUEIROZ, Marcos S. O enfermeiro e a construção da autonomia profissional no processo de cuidar. Revista Brasileira 
de Enfermagem, Brasília, v. 59, n. 2, p. 222-227, abr. 2006. Disponível em: <http://www.scielo. br/scielo.php?script=sci_arttext\&pid=S003471672006000200019\&lng=en\&nrm=iso\&tln $\mathrm{g}=\mathrm{pt}>$. Acesso em: 31 maio 2016.

CAMPOS, Gastão W. D. S.; DOMITTI, Ana C. Apoio matricial e equipe de referência: uma metodologia para gestão do trabalho interdisciplinar em saúde. Cadernos de Saúde Pública, Rio de Janeiro, v. 23, n. 2, p. 399-407, 2007. Disponível em: <http://www.scielo.br/pdf/ csp/v23n2/16.pdf.> Acesso em: 25 out. 2017.

CHANG, Heewon; NGUNJIRI, Faith W.; HERNANDEZ, Kathy-Ann C. Collaborative autoethnography. California: Left Coast Press, 2013.

CIPOLLE, Robert J.; STRAND, Linda M.; MORLEY, Peter C. Pharmaceutical care practice: the patient-centered approach to medication management services. 3. ed. Minnesota: McGraw Hill, 2012.

CONSELHO FEDERAL DE FARMÁCIA (CFF). Resolução n. 586, de 29 de agosto de 2013. p. 1-12. Disponível em: < http://www.cff.org. br/userfiles/file/noticias/Resolução586_13. pdf $>$. Acesso em: 25 out. 2017.

CRESWELL, John W. Qualitative inquiry and research design: choosing among five approaches. 3. ed. Nebraska: Thousand Oaks, 2013.

DETONI, Kirla B. et al. Impact of a medication therapy management service on the clinical status of patients with chronic obstructive pulmonary disease. International Journal of Clinical Pharmacy, Zuidlaren, v. 39, n. 1, p. 95-103, fev. 2017. Disponível em: <http:// www.ncbi.nlm.nih.gov/pubmed/27915426>. Acesso em: 12 fev. 2017.

DUGAN, DeeAnn B. Enhancing community pharmacy through advanced pharmacy practice experiences. American Journal of Pharmaceutical Education, Biddeford, v. 70, n. 1, p. 1-4, 2006.
ELLIS, Carolyn; ADAMS, Tony E.; BOCHNER, Arthur P. Autoethnography: an overview. Forum: Qualitative Social Research, Berlin, v. 12, n. 1, p. 1-23, 2011.

ELROD, Shara et al. Practice change in community pharmacy: a case study of multiple stakeholders' perspectives. Innovations in Pharmacy, Minnesota, v. 3, n. 3, p. 1-6, 2012.

GASTELURRUTIA, Miguel A. et al. Facilitators for practice change in Spanish community pharmacy. Pharmacy World and Science, Zuidlaren, v. 31, n. 1, p. 32-39, fev. 2009. Disponível em: <http://www.ncbi.nlm.nih.gov/ pubmed/18998235> . Acesso em: 23 maio 2016.

GIBERSON, Scott; YODER, Sherri; LEE, Michael P. Improving patient and health system outcomes through advanced pharmacy practice: a report to the US surgeon general. Washington: US Public Health Service, 2011. Disponível em: $<$ https://www.accp.com/docs/positions/misc/ improving_patient_and_health_system_outcomes.pdf>. Acesso em: 25 out. 2017.

HEPLER, Charles D.; STRAND, Linda M. Opportunities and responsibilities in pharmaceutical care. American Journal of Hospital Pharmacy, Maryland, v. 47, n. 3, p. 533-543, 1990.

HIRSCH, Jan D. et al. Primary care-based, pharmacist-physician collaborative medication-therapy management of hypertension: a randomized, pragmatic trial. Clinical Therapeutics, New York, v. 36, n. 9, p. 1.244-1.254, 2014. Disponível em: <https://www.ncbi.nlm. nih.gov/pmc/articles/PMC4169745/> . Acesso em: 25 out. 2017.

ISETTS, Brian J. et al. Clinical and economic outcomes of medication therapy management services: the Minnesota experience. Journal of the American Pharmacists Association, New York, v. 48, n. 2, p. 203-211, 2008.

JONES, Stacy Holman. Autoethnography: making the personal political. In: DENZIN, 
Norman K.; LINCOLN, Yvonna S. (orgs.). Handbook of qualitative research. Thousand Oaks, CA: Sage, 2005. p. 763-791.

McINNIS, Terry; STRAND, Linda M.; WEBB, Edwin C. The patient-centered medical home: integrating comprehensive medication management to optimize patient outcomes. 2. ed. Washington: Patient-Centered Primary Care Collaborative, 2012. Disponível em: https://www.pcpcc.org/sites/default/files/ media/medmanagement.pdf $>$. Acesso em: 25 out. 2017.

MENDES, Eugênio V. O cuidado das condições crônicas na atenção primária à saúde: o imperativo da consolidação da Estratégia Saúde da Família. Brasília: Organização Pan-Americana da Saúde, 2012. Disponível em: < http://bvsms. saude.gov.br/bvs/publicacoes/cuidado_condicoes_atencao_primaria_saude.pdf $>$. Acesso em: 25 out. 2017.

MENDONÇA, Simone A. M. et al. Clinical outcomes of medication therapy management services in primary health care. Brazilian Journal of Pharmaceutical Sciences, São Paulo, v. 52, n. 3, p. 365-373, 2016.

MENDONÇA, Simone A. M.; FREITAS, Erika L.; OLIVEIRA, Djenane R. Competencies for the provision of comprehensive medication management services in an experiential learning project. Plos One, New York, v. 12, n. 9, 2017. Disponível em: <https?doi.org/10.1371/journal.pone. $0185415>$. Acesso em: 25 out. 2017.

MINAYO, Maria Cecília S. Pesquisa social: teoria, método e criatividade. 29. ed. Petrópolis: Vozes, 2010.

MOURÃO, Aline O. M. et al. Pharmaceutical care program for type 2 diabetes patients in Brazil: a randomised controlled trial. International Journal of Clinical Pharmacy, Zuidlaren, v. 35, n. 1, p. 79-86, 2013.

MULHALL, Anne. In the field: notes on observation in qualitative research. Journal of Advanced Nursing, Medford, v. 41, n. 3, p. 306-313, 2003.
NAKAMURA, Carina A.; LEITE, Silvana N. Pharmaceutical services in family health support team: the Brazilian experience. Latin American Journal of Pharmacy, Buenos Aires, v. 34, n. 3, p. 598-601, 2014.

NAKAMURA, Carina A.; LEITE, Silvana N. A construção do processo de trabalho no Núcleo de Apoio à Saúde da Família: a experiência dos farmacêuticos em um município do sul do Brasil. Ciência \& Saúde Coletiva, Rio de Janeiro, v. 21, n. 5, p. 1.565-1.572, 2016. Disponível em: <http://www.scielo.br/pdf/ csc/v2ln5/1413-8123-csc-21-05-1565.pdf>. Acesso em: 25 out. 2017.

OBRELI-NETO, Paulo R. et al. Economic evaluation of a pharmaceutical care program for elderly diabetic and hypertensive patients in primary health care: a 36-month randomized controlled clinical trial. Journal of Managed Care \& Specialty Pharmacy, Alexandria, v. 21, n. 1, p. 66-75, 2015. Disponível em: <http://www.jmcp.org/doi/pdf/10.18553/ jmcp.2015.21.1.66>. Acesso em: 25 out. 2017.

OLIVEIRA, André B. et al. Obstáculos da atenção farmacêutica no Brasil. Revista Brasileira de Ciências Farmacêuticas, São Paulo, v. 41, n. 4, p. 409-413, 2005.

OLIVEIRA, Djenane R. Pharmaceutical care uncovered: an ethnographic study of pharmaceutical care practice. 2003. $393 \mathrm{f}$ (PhD thesis) - University of Minnesota, Minnesota, 2003.

OLIVEIRA, Djenane R.; BRUMMEL, Amanda R.; MILLER, David B. Medication therapy management: 10 years of experience in a large integrated health care system. Journal of Managed Care Pharmacy, Alexandria, v. 16, n. 3, p. 185-195, 2010.

OLIVEIRA, Djenane R. Atenção farmacêutica: da filosofia ao gerenciamento da terapia medicamentosa, compreendendo o conceito, humanizando o cuidado e revolucionando a prática. São Paulo: RCN Editora, 2011.

OLIVEIRA, Djenane R. Brazil. In: CIPOLLE, Robert J.; STRAND, Linda M.; MORLEY, Peter 
C. Pharmaceutical care practice: the patientcentered approach to medication management services. 3. ed. New York: McGraw-Hill, 2012. p. 596-609.

OLIVEIRA, Grazielli C. B. et al. Experiências exitosas de farmacêuticos no SUS. Conselho Federal de Farmácia, Brasília, v. 3, n. 3, p. 71-77, jul. 2015.

OLIVEIRA, Isabela V. et al. Use of the patient's medication experience in pharmacists' decision making process. International Journal of Pharmacy, Bhopa, v. 7, n. 1, p. 1-8, 2017. Disponível em: < http://pharmascholars.com/ PSL/Issue $/ 825 /$ ?title $=$ Use $\% 20$ of $\% 20$ the $\% 20$ patient $\%$ E2 $\% 80 \% 99 \mathrm{~s} \% 20$ medication $\% 20$ experience $\% 20 \mathrm{in} \% 20$ pharmacists $\%$ E2 $\% 80 \% 99 \% 20$ decision $\% 20$ making $\% 20$ process $>$. Acesso em: 13 abr. 2017.

ROBERTS, Alison S. et al. Understanding practice change in community pharmacy: a qualitative study in Australia. Research in Social and Administrative Pharmacy, New York, v. 1, n. 4, p. 546-564, 2005.

SAAR, Sandra R. C.; TREVIZAN, Maria A. Papéis profissionais de uma equipe de saúde: visão de seus integrantes. Revista LatinoAmericana de Enfermagem, Ribeirão Preto, v. 15, n. 1, p.106-12, 2007. Disponível em: <http://www.revistas.usp.br/rlae/article/ view/2404/2674>. Acesso em: 25 out. 2017.

SANDBERG, Jorgen. How do we justify knowledge produced within interpretive approa- ches? Organizational Research Methods, State College, v. 8, n. 1, p. 41-68, 2005.

SECOLI, Silvia R. Polifarmácia: interações e reações adversas no uso de medicamentos por idosos. Revista Brasileira de Enfermagem, Brasília, v. 63, n. 1, p. 136-140, 2010.

SILVA, Danielle F. et al. A extensão universitária como caminho para a construção de serviço de gerenciamento da terapia medicamentosa na atenção primária à saúde. Revista Brasileira de Farmácia Hospitalar e Serviços de Saúde, São Paulo, v. 7, n. 2, p. 15-21, 2016.

SIR, Sumia et al. Barriers for implementation of pharmaceutical care practice in community pharmacies in Sudan. Khartoum Medical Journal, Khartoum, v. 7, n. 1, p. 921-931, 2014.

SORENSEN, Todd D. et al. A qualitative evaluation of medication management services in six Minnesota health systems. American Journal of Health-System Pharmacy, Maryland, v. 73, n. 5, p. 307-314, mar. 2016. Disponível em: <http://www.ajhp.org/cgi/doi/10.2146/ ajhp150212>. Acesso em: 17 maio 2016.

UEMA, Sonia A. et al. Barriers to pharmaceutical care in Argentina. Pharmacy World and Science, Zuidlaren, v. 30, n. 3, p. 211-215, 2008.

Recebido em 03/10/2016. Aprovado em 30/03/2017. 